\title{
The spatial coherence of noise fields evoked by continuous source distributions
}

M. Buerger, T. D. Abhayapala, C. Hofmann, H. Chen, and W. Kellermann

Citation: The Journal of the Acoustical Society of America 142, 3025 (2017); doi: 10.1121/1.5009451

View online: https://doi.org/10.1121/1.5009451

View Table of Contents: https://asa.scitation.org/toc/jas/142/5

Published by the Acoustical Society of America

\section{ARTICLES YOU MAY BE INTERESTED IN}

Acoustic reciprocity: An extension to spherical harmonics domain

The Journal of the Acoustical Society of America 142, EL337 (2017); https://doi.org/10.1121/1.5002078

Evaluation and calibration of mobile phones for noise monitoring application

The Journal of the Acoustical Society of America 142, 3084 (2017); https://doi.org/10.1121/1.5009448

Ambient noise forecasting with a large acoustic array in a complex shallow water environment

The Journal of the Acoustical Society of America 142, EL473 (2017); https://doi.org/10.1121/1.5010764

Comparison of a target-equalization-cancellation approach and a localization approach to source separation

The Journal of the Acoustical Society of America 142, 2933 (2017); https://doi.org/10.1121/1.5009763

Multilevel modeling and regression of community annoyance to transportation noise

The Journal of the Acoustical Society of America 142, 2905 (2017); https://doi.org/10.1121/1.5009581

Wind noise spectra in small Reynolds number turbulent flows

The Journal of the Acoustical Society of America 142, 3227 (2017); https://doi.org/10.1121/1.5012740 


\title{
The spatial coherence of noise fields evoked by continuous source distributions
}

\author{
M. Buerger, ${ }^{1, a)}$ T. D. Abhayapala, ${ }^{2}$ C. Hofmann, ${ }^{1}$ H. Chen, ${ }^{2}$ and W. Kellermann ${ }^{1}$ \\ ${ }^{1}$ Chair of Multimedia Communications and Signal Processing, Friedrich-Alexander-Universität \\ Erlangen-Nürnberg (FAU), Cauerstraße 7, 91058 Erlangen, Germany \\ ${ }^{2}$ Research School of Engineering, College of Engineering and Computer Science, The Australian National \\ University, Canberra, Australian Capital Territory 2601, Australia
}

(Received 11 May 2017; revised 9 October 2017; accepted 11 October 2017; published online 16 November 2017)

In this work, analytic expressions for the spatial coherence of noise fields are derived in the modal domain with the aim of providing a sparse representation. For this purpose, the sound field in a region of interest is expressed in terms of a given pressure distribution on a virtual surrounding cylindrical or spherical surface. According to the Huygens-Fresnel principle, the sound pressure on this surface is represented by a continuous distribution of elementary line or point sources, where orthogonal basis functions characterize the spatial properties. To describe spatially windowed pressure distributions with arbitrary angular extensions, orthogonal basis functions of limited angular support are proposed. As special cases, circular and spherical pressure distributions with uncorrelated source modes of equal power are investigated. It is shown that these distributions result, respectively, in cylindrically isotropic and spherically isotropic, i.e., diffuse noise fields. The analytic expressions derived in this work allow for a prediction of the spatial coherence between arbitrary positions within the region of interest, such that no microphones need to be placed at the actual points of interest. Simulation results are presented to validate the derived relations.

(C) 2017 Acoustical Society of America. https://doi.org/10.1121/1.5009451

[PG]

Pages: $3025-3034$

\section{INTRODUCTION}

Coherence is a property which matters for a large variety of applications and technical areas. First of all, it helps to characterize waves of any kind, be it acoustic waves or electromagnetic waves such as light or radio waves. It plays an important role in mobile communication, ${ }^{1,2}$ in many different multichannel signal processing concepts, e.g., in beamforming, ${ }^{3}$ blind source separation, ${ }^{4,5}$ or dereverberation of acoustic signals, ${ }^{6-8}$ and it is even utilized in certain medical applications, such as medical imaging. ${ }^{9}$ A high spatial coherence is also necessary for a successful deployment of active noise control (ANC) systems, i.e., for canceling acoustic noise in spatially extended areas by emitting "anti-sound." "10-16

When it comes to the quantification of the spatial coherence in practice, the environment and reverberation play a decisive role. Several publications have been dedicated to the coherence estimation for reverberant sound fields, e.g., Refs. 17-19, and references therein. A common assumption is that especially late reverberation affects the estimates of the spatial coherence in a similar way as diffuse sound, which can be approximated by superimposing plane waves of equal amplitude and uniformly distributed random phase originating from all directions. ${ }^{20}$ For this reason, diffuse noise is often considered as one ingredient for modeling complex and reverberant sound fields. ${ }^{21,22}$

In this contribution, the spatial coherence is analytically expressed as a function of a given pressure distribution

\footnotetext{
${ }^{a)}$ Electronic mail: michael.buerger@FAU.de
}

defined on a virtual cylindrical or spherical surface surrounding the region of interest. This pressure distribution may generally be evoked by an arbitrary number of noise sources, but it can always be represented according to the Huygens-Fresnel principle, i.e., by means of a continuous distribution of elementary line or point sources on the surface. The motivation for analyzing continuous source distributions stems from the fact that many real-world noise sources, e.g., a power transformer, an air conditioning system, or complex noise passing through a window, are spatially extended and not point-like. With the aim of providing a compact representation, the source distributions are characterized by so-called source or excitation modes, similar to the work presented by $\mathrm{Wu}$ and Abhayapala. ${ }^{23}$ In this work, the concept of the aforementioned reference is extended and a potential cross-correlation between the individual modes is taken into account. Furthermore, windowed source distributions of arbitrary angular extensions are analyzed. The approach outlined above should provide a means to predict the spatial coherence for any pair of positions within the region of interest, while no sensors are required at the points of interest. In addition, it should give insights into the properties of real sources-it was already shown ${ }^{24,25}$ that vibrating surfaces such as the diaphragm of a loudspeaker can be well described using spatial basis functions.

The remainder of this paper is structured as follows. In Sec. II, the problem tackled in this work is stated and general definitions are formulated. Section III is dedicated to cylindrical surfaces covered with a continuous distribution of elementary line sources, where a relation between the 
cross-correlation of the source modes and the resulting spatial coherence of the evoked 2D variant sound field is derived. A spherical distribution of elementary point sources is considered in Sec. IV, and the correlation of the source modes is related to the spatial coherence of the resulting $3 \mathrm{D}$ variant sound field. The theoretical findings and derived analytic expressions for the spatial coherence are verified by simulations in Sec. V before the work is concluded in Sec. VI.

\section{PROBLEM FORMULATION AND DEFINITIONS}

The spatial coherence between the signals observed at positions $\vec{x}_{\mu}$ and $\vec{x}_{\nu}$ is defined as

$$
\Gamma_{\vec{x}_{\mu} \vec{x}_{\nu}}(\omega)=\frac{\Phi_{\vec{x}_{\mu} \vec{x}_{\nu}}(\omega)}{\sqrt{\Phi_{\vec{x}_{\mu} \vec{x}_{\mu}}(\omega) \Phi_{\vec{x}_{\nu} \vec{x}_{\nu}}(\omega)}}
$$

where $\omega=2 \pi f$ denotes the angular frequency, $\Phi_{\vec{x}_{\mu} \vec{x}_{\nu}}$ is the cross-power spectral density, and $\Phi_{\vec{x}_{\mu} \vec{x}_{\mu}}$ and $\Phi_{\vec{x}_{\nu} \vec{x}_{\nu}}$ are the auto-power spectral densities. In this work, it is assumed that all signals can be characterized as ergodic random processes. The power spectral densities (PSDs) in (1) can then be obtained according to

$$
\Phi_{\vec{x}_{\mu} \vec{x}_{\nu}}(\omega)=\mathcal{E}\left\{P\left(\vec{x}_{\mu}, t, \omega\right) P^{*}\left(\vec{x}_{\nu}, t, \omega\right)\right\},
$$

where $P$ represents the sound pressures at the observation points, $\mathcal{E}\{\cdot\}$ is the expectation operator with respect to (w.r.t.) time, $t$ indicates a time frame of the short-time Fourier transform (STFT), and $(\cdot)^{*}$ denotes complex conjugation.

The aim of this work is to quantify the spatial coherence between an arbitrary pair of observation points located in a sound field evoked by spatially extended noise sources. For this purpose, the investigations of $\operatorname{Cox}^{26}$ serve as a starting point, where the sound field was assumed to be a superposition of uncorrelated plane waves originating from arbitrary directions. We extend this work to sound fields evoked by a continuous distribution of elementary line or point sources and, furthermore, introduce a modal description which considers a potential correlation between the individual elementary sources. As a special case, continuous distributions of line sources with limited angular extension are treated. The presented analysis could be beneficial for efficient implementations of various algorithms, e.g., in the field of ANC, notably if exploiting a sparse representation of the sound field in the modal domain. ${ }^{27,28}$

Throughout this work, all signals are assumed to have a harmonic structure with a time dependency of $\exp (+i \omega t)$, and the forward Fourier transform of a function $f(t)$ is defined as

$$
F(\omega)=\int_{-\infty}^{\infty} f(t) \mathrm{e}^{-i \omega t} \mathrm{~d} t .
$$

We stress that different definitions ${ }^{29}$ are used especially in the acoustics community, which would change, e.g., the formulations of the 2D and 3D Green's functions in Eqs. (5) and (25), respectively.

\section{SPATIAL COHERENCE FOR CONTINUOUS DISTRIBUTION OF LINE SOURCES EVOKING 2D VARIANT SOUND FIELDS}

\section{A. Modal description of the cross-power spectral den- sity in 2D}

Let us consider a planar region of interest $\mathcal{R}$ which is located in the $x-y$ plane and surrounded by a closed contour $\mathcal{Y}$. This contour accommodates a continuous distribution of infinitely long line sources extending along the $z$ axis. Since the line sources are oriented perpendicularly w.r.t. to the $x-y$ plane, the resulting sound field is $z$-invariant. The sound pressure $P$ at any position $\vec{x} \in \mathcal{R}$ in the 2D region of interest can be expressed as

$$
P(\vec{x}, t, \omega)=\oint_{\mathcal{Y}} G(\vec{x} \mid \vec{y}, k) S(\vec{y}, t, \omega) \mathrm{d} \vec{y},
$$

where $S$ characterizes the noise source in the STFT domain at a particular position $\vec{y} \in \mathcal{Y}$ on the contour, $k=\omega / c$ is the wave number, and $c$ denotes the speed of sound. Assuming free-field propagation, the transfer function between $\vec{y}$ and $\vec{x}$ is given by the 2D Green's function ${ }^{30}$

$$
G(\vec{x} \mid \vec{y}, k)=-\frac{i}{4} H_{0}^{(2)}\left(k\|\vec{y}-\vec{x}\|_{2}\right),
$$

where $H_{0}^{(2)}$ is the Hankel function of the second kind and zeroth order, and $\|\cdot\|_{2}$ denotes the Euclidean norm. Substituting Eq. (4) into Eq. (2), the cross-power spectral density $\Phi_{\vec{x}_{1} \vec{x}_{2}}$ for two observation points $\vec{x}_{1}$ and $\vec{x}_{2}$ can be expressed as

$$
\begin{aligned}
\Phi_{\vec{x}_{1} \vec{x}_{2}}(\omega)= & \mathcal{E}\left\{P\left(\vec{x}_{1}, t, \omega\right) P^{*}\left(\vec{x}_{2}, t, \omega\right)\right\} \\
= & \oint_{\mathcal{Y}} \oint_{\mathcal{Y}} G\left(\vec{x}_{1} \mid \vec{y}, k\right) G^{*}\left(\vec{x}_{2} \mid \vec{y}^{\prime}, k\right) \\
& \times \mathcal{E}\left\{S(\vec{y}, t, \omega) S^{*}\left(\vec{y}^{\prime}, t, \omega\right)\right\} \mathrm{d} \vec{y} \mathrm{~d} \vec{y}^{\prime} .
\end{aligned}
$$

If all points $\vec{y}$ of the source distribution lie on a circle with radius $R=\|\vec{y}\|_{2}$ centered at the origin of the coordinate system, they can be more conveniently represented by their angles $\phi_{y}=\angle \vec{y}$ w.r.t. the $x$ axis. This allows us to describe a noise source $S_{R}$ distributed along a circular contour $\mathcal{Y}$ with radius $R$ using a Fourier series expansion

$$
S_{R}\left(\phi_{y}, t, \omega\right)=\sum_{m=-\infty}^{\infty} \beta_{m}(t, \omega) \mathrm{e}^{i m \phi_{y}},
$$

where $\beta_{m}$ are the weights of the basis functions $\exp \left(i m \phi_{y}\right)$, which are referred to as excitation or source modes here. In order to separate the variables $\vec{x}$ and $\vec{y}$ in the argument of the Hankel function in Eq. (5), which is helpful for the integration in Eqs. (4), (6), we apply Graf's addition theorem, ${ }^{31}$

$$
\begin{aligned}
H_{0}^{(2)} & \left(k\|\vec{y}-\vec{x}\|_{2}\right) \\
& =\sum_{n=-\infty}^{\infty} H_{n}^{(2)}(k R) J_{n}(k X) \mathrm{e}^{i n\left(\phi_{x}-\phi_{y}\right)} \quad \text { for } \quad X<R,
\end{aligned}
$$

where $X=\|\vec{x}\|_{2}$ and $\phi_{x}=\angle \vec{x}$. Furthermore, $J_{n}$ and $H_{n}^{(2)}$ denote the $n$th order Bessel function and Hankel function of 
the second kind, respectively. In the remainder of this paper, we always assume that the condition $X_{i}<R$, with $X_{i}=\left\|\vec{x}_{i}\right\|_{2}$ and $i \in\{1,2\}$, is met. Introducing the cross-correlation $\rho_{m m^{\prime}}(\omega)=\mathcal{E}\left\{\beta_{m}(t, \omega) \beta_{m^{\prime}}^{*}(t, \omega)\right\}$ of the weights for the excitation modes and substituting Eqs. (5), (7), and (8) into Eq. (6) yields

$$
\begin{aligned}
\stackrel{\circ}{\Phi}_{\vec{x}_{1} \vec{x}_{2}}(\omega)= & \frac{1}{16} \oint_{\mathcal{Y}} \oint_{\mathcal{Y}_{m, m^{\prime}, n, n^{\prime}}=-\infty} \sum_{n}^{\infty} H_{n}^{(2)}(k R) J_{n}\left(k X_{1}\right) H_{n^{\prime}}^{(1)}(k R) \\
& \times J_{n^{\prime}}\left(k X_{2}\right) \rho_{m m^{\prime}}(\omega) \mathrm{e}^{i n\left(\phi_{x_{1}}-\phi_{y}\right)} \mathrm{e}^{-i n^{\prime}\left(\phi_{x_{2}}-\phi_{y}^{\prime}\right)} \\
& \times \mathrm{e}^{i\left(m \phi_{y}-m^{\prime} \phi_{y}^{\prime}\right)} \mathrm{d} \phi_{y} \mathrm{~d} \phi_{y}^{\prime},
\end{aligned}
$$

where the circle above $\Phi_{\vec{x}_{1} \vec{x}_{2}}$ indicates that the integration is performed over a circular contour in the angular interval $[0,2 \pi)$, and $H_{n^{\prime}}^{(1)}=H_{n^{\prime}}^{(2)} *$ is the $n^{\prime}$ th order Hankel function of the first kind. The auto-power spectral densities $\Phi_{\vec{x}_{1} \vec{x}_{1}}$ and $\Phi_{\vec{x}_{2} \vec{x}_{2}}$ can be computed analogously to Eq. (9) such that the spatial coherence can eventually be obtained according to Eq. (1).

Due to the orthogonality of the complex exponential functions, the expression for the cross-power spectral density in (9) significantly simplifies according to

$$
\begin{aligned}
{\stackrel{\circ}{\Phi_{\vec{x}_{1} \vec{x}_{2}}}}_{(\omega)=} & \frac{\pi^{2}}{4} \sum_{n, n^{\prime}=-\infty}^{\infty} H_{n}^{(2)}(k R) J_{n}\left(k X_{1}\right) H_{n^{\prime}}^{(1)}(k R) J_{n^{\prime}}\left(k X_{2}\right) \\
& \times \rho_{-n-n^{\prime}}(\omega) \mathrm{e}^{i\left(n \phi_{x_{1}}-n^{\prime} \phi_{x_{2}}\right)} .
\end{aligned}
$$

In practice, the number of modes utilized to describe the true sound field is usually truncated while still retaining a sufficient accuracy. Given that the area of interest $\mathcal{R}$ has a radius of $r_{\max }$, the summation in Eq. (10) can be limited to $n, n^{\prime}=-N, \ldots, N$, where $^{32}$

$$
N=\left\lceil\frac{1}{2} \mathrm{e} r_{\max } k_{\max }\right\rceil
$$

with $k_{\max }=\omega_{\max } / c$, the ceiling operator $\lceil\cdot\rceil$, and e being Euler's number. Choosing this number assures that the approximation error of the sound field within $\mathcal{R}$ will be below $16.1 \%$ for angular frequencies up to $\omega_{\max }{ }^{32}$

\section{B. Cylindrically isotropic noise field}

Let us consider the special case of uncorrelated source modes with unit power for all frequencies $\omega$. In this case, $\rho_{-n-n^{\prime}}(\omega)=\delta_{n n^{\prime}}$, with $\delta_{n n^{\prime}}$ representing the Kronecker delta, and Eq. (10) reduces to

$$
\begin{aligned}
{\stackrel{\text { o uncorr }}{\Phi_{\vec{x}_{1} \vec{x}_{2}}}}_{(\omega)=} & \frac{\pi^{2}}{4} \sum_{n=-\infty}^{\infty}\left|H_{n}(k R)^{(2)}\right|^{2} J_{n}\left(k X_{1}\right) J_{n}\left(k X_{2}\right) \\
& \times \mathrm{e}^{i n\left(\phi_{x_{1}}-\phi_{x_{2}}\right) .}
\end{aligned}
$$

If we furthermore assume the far-field case with $k R \gg 1$, we can exploit the large-argument approximation of the Hankel function $^{33}$ and approximate Eq. (12) according to

$$
\begin{aligned}
\underset{{\stackrel{x}{x_{1}}}_{\vec{x}_{2}}}{\text { uncorr }}(\omega) & \approx \frac{\pi}{2 k R} \sum_{n=-\infty}^{\infty} J_{n}\left(k X_{1}\right) J_{n}\left(k X_{2}\right) \mathrm{e}^{i n\left(\phi_{x_{1}}-\phi_{x_{2}}\right)} \\
& =\frac{\pi}{2 k R} J_{0}(k \Delta X),
\end{aligned}
$$

where $\Delta X=\left\|\vec{x}_{1}-\vec{x}_{2}\right\|_{2}$ and Graf's addition theorem for the Bessel function ${ }^{33}$ is exploited to obtain Eq. (14) from Eq. (13). After computing the auto-power spectral densities at $\vec{x}_{1}$ and $\vec{x}_{2}$ according to Eq. (14), the spatial coherence in Eq. (1) results in the well-known coherence for a cylindrically isotropic noise field, ${ }^{34}$

$$
\Gamma_{\vec{x}_{1} \vec{x}_{2}}^{\text {iso }}(\omega)=J_{0}(k \Delta X) .
$$

That is, a continuous distribution of line sources located on a circular contour with a large radius results in an isotropic noise field inside the contour if all source modes are mutually uncorrelated and the corresponding weights are of equal power.

\section{Sources of limited angular extension}

Next, we consider the case where the sound source does not surround the area of interest completely, i.e., $\mathcal{Y}$ is not a closed contour, but an arc with angular extension $\Delta \phi_{y}$. The resulting cross-power spectral densities can again be obtained from Eq. (9), where the integration must be limited to the desired interval. Without loss of generality, we consider the source distribution to be centered at $\phi_{y}=0^{\circ}$ such that the source extends in the interval $\left[-\Delta \phi_{y} / 2, \Delta \phi_{y} / 2\right]$. This limitation of the source extension can be described by a multiplication of the sound pressure $S_{R}$ on the contour with a rectangular window function in the angular domain. After performing this windowing operation and the integration in Eq. (9), we obtain

$$
\begin{aligned}
\breve{\Phi}_{\vec{x}_{1} \vec{x}_{2}}(\omega) & \\
= & \frac{1}{16}\left(\Delta \phi_{y}\right)^{2} \sum_{n, n^{\prime}=-\infty}^{\infty} H_{n}^{(2)}(k R) J_{n}\left(k X_{1}\right) H_{n^{\prime}}^{(1)}(k R) \\
& \times J_{n^{\prime}}\left(k X_{2}\right) \mathrm{e}^{i\left(n \phi_{x_{1}}-n^{\prime} \phi_{x_{2}}\right)} \\
& \times \sum_{m^{\prime}=-\infty}^{\infty} \underbrace{\sum_{m=-\infty}^{\infty} \rho_{m m^{\prime}}(\omega) \operatorname{sinc}\left(\frac{n+m}{2} \Delta \phi_{y}\right)}_{\text {discrete convolution w.r.t. } m} \\
& \times \operatorname{sinc}\left(\frac{n^{\prime}+m^{\prime}}{2} \Delta \phi_{y}\right),
\end{aligned}
$$

where $\operatorname{sinc}(\xi)=\sin (\xi) / \xi$. An inspection of Eq. (16) reveals that a multiplication with a rect-function in the angular domain results in a discrete convolution of $\rho_{\mathrm{mm}}$ with a sinc-function in the modal domain. Again, the number of modes utilized to describe the sound field can be limited according to Eq. (11). However, due to the involved convolutions, source modes $m, m^{\prime}>N$ will also have an impact on lower-order modes $n, n^{\prime} \leq N$. This implies that the modal truncation can only be done after performing the convolution. To realize the sinc-function in practice, a window with a suitably chosen length needs to be applied such that the approximation error can be neglected. It is worth noting here that for the special case $\Delta \phi_{y}=2 \pi$, the sinc-functions will always be zero except for $m=-n$ and $m^{\prime}=-n^{\prime}$ leading to a value of 1 , which means that Eq. (16) becomes identical to Eq. (10). 


\section{Sources of limited angular extension represented by alternative basis functions}

In order to precisely describe the rectangular function for windowed sources (see Sec. III C) by means of a Fourier series, theoretically infinitely many basis functions are required. It is thus desirable to utilize a set of orthogonal basis functions $B_{m}$ with limited angular support, i.e., basis functions which are nonzero only within the limited angular region of extension $\Delta \phi_{y}$ and zero elsewhere. A straightforward approach would be the use of windowed complex exponential functions,

$$
B_{m}\left(\phi_{y}\right)=\operatorname{rect}\left(\frac{\phi_{y}}{\Delta \phi_{y}}\right) \mathrm{e}^{i m\left(\phi_{y} / \Delta \phi_{y}\right) 2 \pi},
$$

the local spatial frequencies of which are integer multiples $m$ of the local spatial fundamental frequency $1 / \Delta \phi_{y}$ determined by the window size. In comparison, the spatial fundamental frequency of the Fourier basis functions is $1 /(2 \pi)$. That is, the local spatial frequencies are scaled by a factor $2 \pi / \Delta \phi_{y}$ relative to the spatial frequencies of the Fourier basis functions. An illustration of the basis functions $B_{m}$ is given in Fig. 1 together with the basis functions used for the Fourier series expansion in Eq. (7). Note that, for simplicity and without loss of generality, the modified basis functions (17) are defined such that they are centered at $\phi_{y}=0^{\circ}$ corresponding to $y=0$. Using Eq. (17), a sound source of angular extension $\Delta \phi_{y}$ can be conveniently described as

$$
S_{R}\left(\phi_{y}, t, \omega\right)=\sum_{m=-\infty}^{\infty} g_{m}(t, \omega) B_{m}\left(\phi_{y}\right)
$$

with $g_{m}$ being the weight of the corresponding basis function. Similar to a Fourier series expansion, the weights $g_{m}$

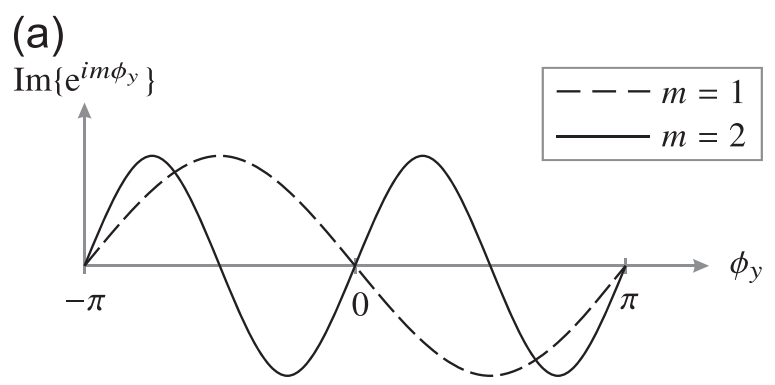

(b)

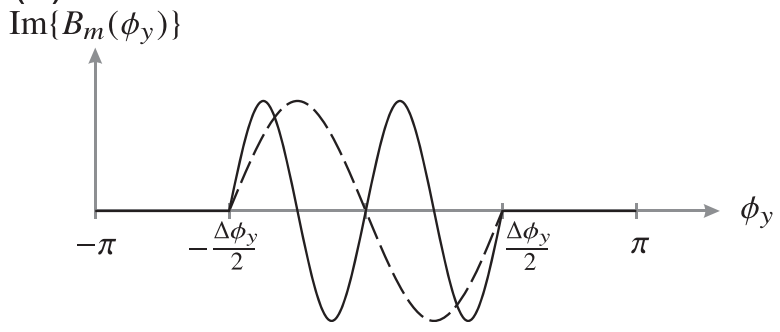

FIG. 1. Illustration of (b) the proposed basis functions $B_{m}$, whose angular support is limited to a range of $\Delta \phi_{y}$, in comparison with (a) the corresponding basis functions of a Fourier series expansion for $m=1,2$. can be computed for a given sound pressure $S_{R}$ by evaluating the inner product

$$
g_{m}(t, \omega)=\frac{1}{\Delta \phi_{y}} \int_{-\Delta \phi_{y} / 2}^{\Delta \phi_{y} / 2} S_{R}\left(\phi_{y}, t, \omega\right) B_{m}^{*}\left(\phi_{y}\right) \mathrm{d} \phi_{y} .
$$

Following the same steps as above, i.e., substituting Eqs. (17), (5), and (8) into Eq. (6) and performing the integration, we can describe the cross-power spectral density for two observation points in an analogous way,

$$
\begin{aligned}
\breve{\Phi}_{\vec{x}_{1} \vec{x}_{2}}(\omega)= & \frac{1}{16}\left(\Delta \phi_{y}\right)^{2} \sum_{m, m^{\prime}, n, n^{\prime}=-\infty}^{\infty} H_{n}^{(2)}(k R) J_{n}\left(k X_{1}\right) \\
& \times H_{n^{\prime}}^{(1)}(k R) J_{n^{\prime}}\left(k X_{2}\right) \breve{\rho}_{m m^{\prime}}(\omega) \\
& \times \operatorname{sinc}\left(n \frac{\Delta \phi_{y}}{2}+m \pi\right) \operatorname{sinc}\left(n^{\prime} \frac{\Delta \phi_{y}}{2}+m^{\prime} \pi\right) \\
& \times \mathrm{e}^{i\left(-n \phi_{x_{1}}+n^{\prime} \phi_{x_{2}}\right)},
\end{aligned}
$$

where $\breve{\rho}_{m m^{\prime}}(\omega)=\mathcal{E}\left\{g_{m}(t, \omega) g_{m^{\prime}}^{*}(t, \omega)\right\}$ denotes the crosscorrelation of the weights for the basis functions $B_{m}$. From Eq. (20) it can be seen that the representation of the crosspower spectral density with the new set of basis functions is similar to the original one in Eq. (16). However, the number of basis functions required to describe an angularly limited noise source can now be significantly reduced. For example, a solid vibrating surface (of infinite height $z$ ) could be described by the zeroth basis function $B_{0}$ only, which means that only the summations over $n$ and $n^{\prime}$ would remain. Even if several basis functions are active, the corresponding arguments $m$ and $m^{\prime}$ of the sinc-functions in Eq. (20) are scaled by $\pi$ rather than $\Delta \phi_{y} / 2$. This implies that, if $\Delta \phi_{y}<2 \pi$, the envelopes of the sinc-functions decay faster relative to Eq. (16), which is especially significant for low values of $\Delta \phi_{y}$, such that shorter windows can be applied to the sincfunctions. For completeness, it should be noted that Eq. (20) also simplifies to Eq. (10) if $\Delta \phi_{y}=2 \pi$.

To further illustrate the benefit of the new basis functions, we now consider an example where the angular extension of the noise source is an integer fraction of the entire circle, i.e., $\Delta \phi_{y}=2 \pi / L$ with $L \in \mathbb{N}^{+}$. Let us assume that $2 \breve{M}+1$ of these basis functions are required in order to resolve a certain maximum spatial frequency within the angular window $\Delta \phi_{y}$. If the same spatial frequency is to be resolved with the original excitation modes defined in Eq. (7) for the entire circle, $2 L M+1$ modes are required. This is due to the fact that the angularly limited basis functions correspond to compressed versions of the original excitation modes and, thus, have an $L$ times larger spatial frequency, which can be seen from Eq. (17). In other words, $L$ times fewer basis functions are required in Eq. (20) in order to model the same maximum spatial frequency as in Eq. (16).

For an actual implementation of Eq. (20), it is necessary to truncate the individual summations. This truncation also allows to simultaneously and conveniently express the crossPSDs for a set of $Q$ observations points using a compact matrix notation. For this purpose, we define a $Q \times 2 N+1$ matrix $[\mathbf{H}(k, \vec{x}, R)]_{\mu, n}=H_{n}^{(2)}(k R) J_{n}\left(k X_{\mu}\right) \mathrm{e}^{i n \phi_{x_{\mu}}}$ governed by 
the source radius $R$ and the positions of the observations points, a $2 N+1 \times 2 \breve{M}+1$ matrix $\left[\breve{\xi}\left(\Delta \phi_{y}\right)\right]_{n, m}=\operatorname{sinc}\left(n\left(\Delta \phi_{y}\right)\right.$ $2)+m \pi)$ governed by the angular extension $\Delta \phi_{y}$ of the source, and a $2 \breve{M}+1 \times 2 \breve{M}+1$ correlation matrix $\left[\breve{\mathbf{R}}_{\mathbf{g g}}(\omega)\right]_{m, m^{\prime}}=\mathcal{E}\left\{g_{m}(t, \omega) g_{m^{\prime}}^{*}(t, \omega)\right\}$ for the weights of the basis functions $B_{m}$. The $Q \times Q$ PSD matrix $\breve{\boldsymbol{\Phi}}$ for the set of $Q$ observation points can then be written as

$$
\breve{\boldsymbol{\Phi}}(\omega)=\mathbf{H}(k, \vec{x}, R) \breve{\xi}\left(\Delta \phi_{y}\right) \breve{\mathbf{R}}_{\mathrm{gg}}(\omega) \breve{\xi}^{\mathrm{T}}\left(\Delta \phi_{y}\right) \mathbf{H}^{\mathrm{H}}(k, \vec{x}, R),
$$

where the superscripts $(\cdot)^{\mathrm{T}}$ and $(\cdot)^{\mathrm{H}}$ denote transposition and conjugate transposition, respectively, and the dependencies on the left-hand side are omitted for the sake of a compact notation.

For completeness, it should also be noted that an alternative way to define a set of orthogonal bases are the Slepian functions which, for the $1 \mathrm{D}$ case, are referred to as prolate spheroidal functions. ${ }^{35,36}$ The advantage of this kind of functions is that they are strictly limited in one domain while being maximally concentrated in the corresponding transform domain: For example, Slepian basis functions based on cylindrical harmonics would maximize the ratio of the energy within an angular window $\Delta \phi_{y}$ and the energy on the full circle, while only a strictly limited number of basis functions is used.

\section{SPATIAL COHERENCE FOR CONTINUOUS DISTRIBUTION OF POINT SOURCES EVOKING 3D VARIANT SOUND FIELDS}

\section{A. Modal description of the cross-power spectral den- sity in 3D}

The sound pressure at observation point $\vec{x}$ for the $3 \mathrm{D}$ case is evoked by a continuous source distribution $S$ defined on an acoustically transparent surface surrounding the region of interest, i.e.,

$$
P(\vec{x}, t, \omega)=\oiint_{\mathcal{A}} \underbrace{\frac{\mathrm{e}^{-i k|| \vec{y}-\vec{x} \|_{2}}}{4 \pi\|\vec{y}-\vec{x}\|_{2}}}_{G^{3 \mathrm{D}}(\vec{x} \mid \vec{y}, k)} S(\vec{y}, t, \omega) \mathrm{d} \vec{y},
$$

where $G^{3 \mathrm{D}}$ is the 3D Green's function ${ }^{30}$ and $\vec{y}$ represents a point on the surface $\mathcal{A}$. Considering a spherical surface, any position $\vec{y} \in \mathcal{A}$ can be parametrized by a constant radius $R$, an elevation angle $\theta_{y}$, and an azimuth angle $\phi_{y}$. The sound pressure $S_{R}$ on this sphere can then be described using spherical harmonics, ${ }^{30}$

$$
S_{R}\left(\theta_{y}, \phi_{y}, t, \omega\right)=\sum_{n=0}^{\infty} \sum_{m=-n}^{n} \alpha_{n}^{m}(t, \omega) Y_{n}^{m}\left(\theta_{y}, \phi_{y}\right),
$$

where $Y_{n}^{m}$ is the spherical harmonic of degree $n$ and order $m$, and $\alpha_{n}^{m}$ denotes the corresponding weight. The spherical harmonics are defined as ${ }^{31}$

$$
Y_{n}^{m}(\theta, \phi)=\sqrt{\frac{2 n+1}{4 \pi} \cdot \frac{(n-|m|) !}{(n+|m|) !}} P_{n}^{|m|}(\cos \theta) \mathrm{e}^{i m \phi}
$$

with $P_{n}^{|m|}$ being the associated Legendre function of degree $n$ and order $m$. After introducing the $n$th order spherical Bessel function $j_{n}$ and spherical Hankel function $h_{n}^{(2)}$ of the second kind, the 3D Green's function can be expanded using the addition theorem, ${ }^{31}$

$$
\begin{aligned}
G^{3 \mathrm{D}}(\vec{x} \mid \vec{y}, k)= & -\sum_{n=0}^{\infty} \sum_{m=-n}^{n} i k h_{n}^{(2)}(k R) Y_{n}^{m *}\left(\theta_{y}, \phi_{y}\right) j_{n}(k X) \\
& \times Y_{n}^{m}\left(\theta_{x}, \phi_{x}\right) .
\end{aligned}
$$

Inserting Eq. (25) together with Eq. (23) into Eq. (22), the cross-power spectral density according to Eq. (2) for two observation points $\vec{x}_{1}$ and $\vec{x}_{2}$ can be written as

$$
\begin{aligned}
\stackrel{\circ}{\Phi}_{\vec{x}_{1} \vec{x}_{2}}^{3 \mathrm{D}}(\omega)= & k^{2} \oiint_{\mathcal{A}} \oiint_{\mathcal{A}} \sum_{p, p^{\prime}, n, n^{\prime}=0}^{\infty} \sum_{q=-p}^{p} \sum_{q^{\prime}=-p^{\prime}}^{p^{\prime}} \sum_{m=-n}^{n} \sum_{m^{\prime}=-n^{\prime}}^{n^{\prime}} h_{p}^{(2)}(k R) Y_{p}^{q *}\left(\theta_{y}, \phi_{y}\right) j_{p}\left(k X_{1}\right) Y_{p}^{q}\left(\theta_{x_{1}}, \phi_{x_{1}}\right) \\
& \times h_{p^{\prime}}^{(1)}(k R) Y_{p^{\prime}}^{q^{\prime}}\left(\theta_{y}^{\prime}, \phi_{y}^{\prime}\right) j_{p^{\prime}}\left(k X_{2}\right) Y_{p^{\prime}}^{q^{\prime} *}\left(\theta_{x_{2}}, \phi_{x_{2}}\right) \rho_{n n^{\prime}}^{m m^{\prime}}(\omega) Y_{n}^{m}\left(\theta_{y}, \phi_{y}\right) Y_{n^{\prime}}^{m^{\prime} *}\left(\theta_{y}^{\prime}, \phi_{y}^{\prime}\right) \\
& \times \sin \theta_{y} \mathrm{~d} \theta_{y} \mathrm{~d} \phi_{y} \sin \theta_{y}^{\prime} \mathrm{d} \theta_{y}^{\prime} \mathrm{d} \phi_{y}^{\prime},
\end{aligned}
$$

where $\rho_{n n^{\prime}}^{m m^{\prime}}(\omega)=\mathcal{E}\left\{\alpha_{n}^{m}(t, \omega) \alpha_{n^{\prime}}^{m^{\prime} *}(t, \omega)\right\}$ represents the crosscorrelation of the weights for the spherical harmonics in Eq. (23), and $h_{p^{\prime}}^{(1)}=h_{p^{\prime}}^{(2) *}$ denotes the $p^{\prime}$ th order spherical Hankel function of the first kind.

\section{B. Spherically isotropic noise field}

Similar to the 2D case, we specifically treat a noise source consisting of uncorrelated modes with unit power, i.e., $\rho_{n n^{\prime}}^{m m^{\prime}}(\omega)=\delta_{n n^{\prime}} \delta_{m m^{\prime}}$. Exploiting the orthogonality of spherical harmonics, the cross-power spectral density in Eq. (26) simplifies according to

$$
\begin{aligned}
& \stackrel{\circ 3 \mathrm{D}, \text { uncorr }}{{\stackrel{\Phi}{\vec{x}_{1} \vec{x}_{2}}}_{\vec{x}^{2}}}(\omega)=k^{2} \sum_{n=0}^{\infty} \sum_{m=-n}^{n}\left|h_{n}(k R)^{(2)}\right|^{2} j_{n}\left(k X_{1}\right) \\
& \times Y_{n}^{m}\left(\theta_{x_{1}}, \phi_{x_{1}}\right) j_{n}\left(k X_{2}\right) Y_{n}^{m *}\left(\theta_{x_{2}}, \phi_{x_{2}}\right) .
\end{aligned}
$$

After utilizing the large-argument approximation of the spherical Hankel functions ${ }^{31}$ for $k R \gg 1$ and applying the addition theorem for the spherical Bessel function, ${ }^{37}$ the cross-power spectral density in Eq. (27) turns into 


$$
\stackrel{\stackrel{3}{\Phi} \vec{x}_{\vec{x}_{1}, \vec{x}_{2}}}{\text { uncorr }}(\omega) \approx \frac{1}{4 \pi R^{2}} j_{0}(k \Delta X) .
$$

Using this result and computing the auto-power spectral densities for $\vec{x}_{1}$ and $\vec{x}_{2}$ accordingly yields

$$
\Gamma_{\vec{x}_{1} \vec{x}_{2}}^{\text {diff }}(\omega)=j_{0}(k \Delta X)=\operatorname{sinc}(k \Delta X) .
$$

The expression in Eq. (29) describes the spatial coherence of a spherically isotropic noise field, which again is a wellknown result ${ }^{34}$ and also referred to as diffuse noise.

\section{SIMULATIONS}

To validate the analytic description of the spatial coherence derived above, we conduct the following simulations: First, a set of discrete line sources located on a circular contour around the region of interest is investigated in Sec. V A. These line sources cover different angular extensions and directly define the sound pressure on the cylindrical surface surrounding the observation region. In the second simulation, presented in Sec. V B, an "open window" scenario is mimicked, where the sound waves created by multiple simultaneously active line sources travel from the outside through an opening into the region of interest. For both scenarios, a sampling frequency $f_{\mathrm{s}}=8 \mathrm{kHz}$ is used, and the spatial coherence at the observation points as obtained with PSDs computed after Eq. (21) is compared to a classical coherence estimation using the Welch method applied to the simulated signals at the actual observation points.

\section{A. Mutually independent line sources on a circular contour}

Let us consider a continuous distribution of line sources located on an arc of angular extension $\Delta \phi_{y}$. This source distribution is approximated by $N_{\mathrm{S}}$ discrete line sources located on the arc with an inter-element spacing of $0.5^{\circ}$, where the number $N_{\mathrm{S}}$ of utilized sources depends on the angular extension $\Delta \phi_{y}$. These line sources emit mutually uncorrelated white Gaussian noise signals of equal power such that the sound pressure at different discrete source positions on the contour is spatially white. A schematic illustration of the considered setup is given in Fig. 2, where the source positions and observation points are represented by solid dots

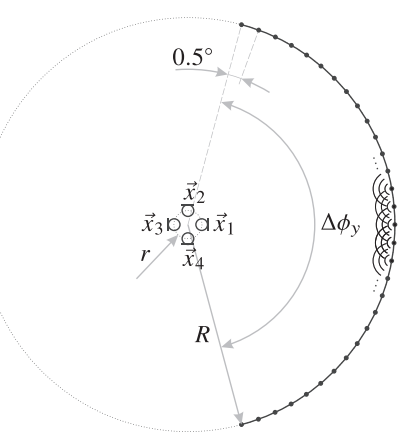

FIG. 2. Illustration of the simulation setup with line sources located on the contour and emitting mutually uncorrelated white Gaussian noise, where the source positions and observation points are indicated by solid dots and microphones, respectively. and microphones, respectively. All observation points are located on a circle of radius $r=0.2 \mathrm{~m}$ centered at the origin, where positions $\vec{x}_{1}$ and $\vec{x}_{3}$ are separated by $\Delta X=0.4 \mathrm{~m}$ along the $x$ axis, and positions $\vec{x}_{2}$ and $\vec{x}_{4}$ are separated by $\Delta Y=0.4 \mathrm{~m}$ along the $y$ axis. The source radius $R$ is chosen as $3 \mathrm{~m}$, and angular extensions $\Delta \phi_{y} \in\left\{20^{\circ}, 45^{\circ}, 60^{\circ}\right.$, $\left.90^{\circ}, 135^{\circ}, 180^{\circ}, 270^{\circ}, 360^{\circ}\right\}$ are considered. It should be noted that, even though the spatial coherence obviously is a function of $k$ and the spacing between the observation points, we consider a fixed spacing and treat the angular extension as a variable here to evaluate the impact of differently-sized sources.

To evaluate the PSD matrix $\breve{\boldsymbol{\Phi}}$ in Eq. (21) for the considered setup, the correlation matrix $\breve{\mathbf{R}}_{\text {gg }}$ for the weights $g_{m}$ is required. However, due to the fact that the signals on the contour are spatially white, the weights $g_{m}$ for the basis functions $B_{m}$ defined in Eq. (17) also need to be mutually uncorrelated and of equal power. This can be seen by investigating the respective correlation matrices: Let $\mathbf{s}(t, \omega)=\left[S\left(\vec{y}_{1}, t, \omega\right)\right.$, $\left.\ldots, S\left(\vec{y}_{N_{\mathrm{S}}}, t, \omega\right)\right]^{\mathrm{T}}$ be a vector capturing the individual source signals and let $\mathbf{R}_{\mathrm{ss}}$ denote the corresponding correlation matrix, which is defined as

$$
\mathbf{R}_{\mathbf{s s}}(\omega)=\mathcal{E}\left\{\mathbf{s}(t, \omega) \mathbf{s}^{\mathrm{H}}(t, \omega)\right\} .
$$

In the case of mutually uncorrelated source signals of unit power, the correlation matrix $\mathbf{R}_{\mathbf{s s}}$ results in an identity matrix. For discrete source positions, the weights $g_{m}$ can be obtained by computing the discrete equivalent of the inner product in Eq. (19), i.e., they are the result of an inverse spatial discrete Fourier transform (DFT) with scaled basis functions Eq. (17). Therefore, the correlation matrix for the weights $g_{m}$ is given by

$$
\breve{\mathbf{R}}_{\mathbf{g g}}(\omega)=\mathbf{F}^{\mathrm{H}}(m, \omega) \mathcal{E}\left\{\mathbf{s}(t, \omega) \mathbf{s}^{\mathrm{H}}(t, \omega)\right\} \mathbf{F}(m, \omega),
$$

where $\mathbf{F}$ represents the corresponding DFT matrix of dimension $N_{\mathrm{S}} \times N_{\mathrm{S}}$. As the DFT matrix is orthonormal, the correlation matrix $\breve{\mathbf{R}}_{\mathrm{gg}}(\omega)$ will also be an identity matrix in case of uncorrelated source signals of unit power, implying that the modal weights are also uncorrelated and of unit power. Consequently, the PSD matrix can be directly evaluated without an estimation of the correlation matrix. Note that an infinite number of basis functions $B_{m}$ would be required in order to obtain a constant power distribution on the entire (continuous) contour, as would be the case for the number of discrete line sources. However, due to the fact that only a limited number of modes have a nonnegligible contribution to the sound field at the observation points, i.e., within the region of interest $\mathcal{R}$ defined by a circle of radius $r_{\max }$, the higher-order basis functions can be neglected. ${ }^{32}$ In practice, the number of observable basis functions is limited by the number of utilized microphones. Future work will consider the required number of basis functions for accurately describing the sound field within $\mathcal{R}$.

The spatial coherence between $\vec{x}_{1}$ and $\vec{x}_{3}$ as obtained with the PSDs computed after Eq. (21) is shown in Fig. 3 for $N=\breve{M}=20$, which is twice the number resulting from Eq. (11) and assures a higher accuracy. For verification purposes, 


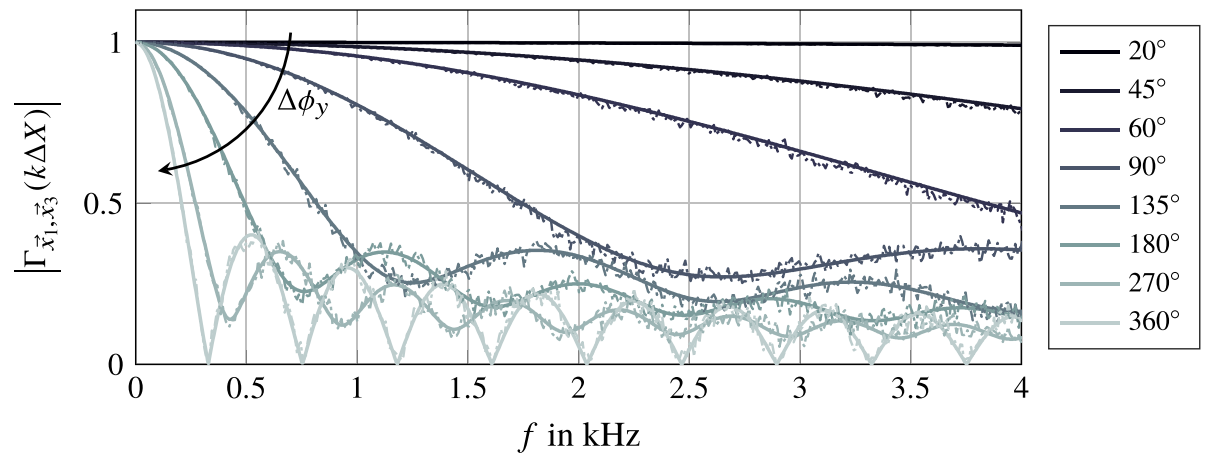

FIG. 3. (Color online) Spatial coherence between a pair of observations points $\vec{x}_{1}$ and $\vec{x}_{3}$ separated by a distance of $\Delta X=0.4 \mathrm{~m}$ along the $x$ axis. The underlying noise field is evoked by a continuous distribution of line sources of different angular extensions $\Delta \phi_{y} \in\left\{20^{\circ}, 45^{\circ}, 60^{\circ}, 90^{\circ}, 135^{\circ}, 180^{\circ}, 270^{\circ}, 360^{\circ}\right\}$ with radius $R=3 \mathrm{~m}$ and uncorrelated weights $g_{m}$ of unit power. The solid lines represent the analytically computed coherence based on Eq. (21), whereas the dashdotted lines are obtained with the Welch method applied to a simulated noise field evoked by a finite number of uncorrelated line sources.

the spatial coherence obtained by applying the Welch method to the simulated time-domain signals at the observation points is also plotted in Fig. 3 with dash-dotted lines. It can be seen that the simulated and the analytically evaluated spatial coherence curves match very well. As already mentioned above, uncorrelated source modes with $\Delta \phi_{y}=360^{\circ}$ result in a cylindrically isotropic noise field corresponding to the zeroth order Bessel function, which can be clearly observed in the plot. For decreasing values of $\Delta \phi_{y}$, the coherence increases on average. This is an expected result, as the continuous source distribution becomes a single line source if $\Delta \phi_{y} \rightarrow 0^{\circ}$, which results in a fully coherent sound field.

We now consider the second pair of observation points $\vec{x}_{2}$ and $\vec{x}_{4}$, which are rotated by $90^{\circ}$ relative to the first pair and separated by $\Delta Y=0.4 \mathrm{~m}$ along the $y$ axis. The resulting spatial coherence with PSDs according to Eq. (21) is shown in Fig. 4 for angular extensions $\Delta \phi_{y} \in\left\{20^{\circ}, 45^{\circ}, 60^{\circ}, 90^{\circ}\right\}$ and compared to the coherence for the first pair of observation points $\vec{x}_{1}$ and $\vec{x}_{3}$. The plot reveals that the spatial coherence values strongly depend on the orientation of the pair of observation points. It can be seen that the spatial coherence is much higher if the connection line between the observation points is parallel to the principal orientation of the sound intensity, i.e., the "main" propagation direction of the sound waves (along the $x$ axis), whereas lower values are obtained if the connection line is oriented perpendicularly. This can be explained with the trace wavenumber $k_{\|}$, i.e., the "effective" wavenumber as observed by a pair of observation points, which is given by the projection of the wavenumber onto the connecting line. For a plane wave incident from direction $\phi_{\mathrm{PW}}$ relative to the connecting line, the trace wavenumber is given by $k_{\|}=k \cos \left(\phi_{\mathrm{PW}}\right)$, implying that the observed wavenumber $k_{\|}$may be much lower than $k$ for some directions. Due to this projection, the orientation of the pair of observation points may have a strong impact on the coherence.

\section{B. "Open window"}

As a second simulation, we mimic a scenario where noise is traveling from the outside through an open window into the region of interest. That is, the sound pressure on the contour is not directly evoked on the same, but it results from four line sources located outside the window, as illustrated in Fig. 5. Uncorrelated white Gaussian noise with variances $\sigma_{1}^{2}=0.04, \sigma_{2}^{2}=0.25, \sigma_{3}^{2}=0.81$, and $\sigma_{4}^{2}=0.16$ are used as individual source signals, and the positions of the respective sources are specified in terms of their $[x, y]$ coordinates: $\vec{y}_{1}=[4,0] \mathrm{m}, \quad \vec{y}_{2}=[6,-2] \mathrm{m}, \quad \vec{y}_{3}=[8,2] \mathrm{m}, \quad$ and $\vec{y}_{4}=[7,-1] \mathrm{m}$. The shaded parts in Fig. 5 can be thought of as perfectly absorbing infinitely long walls such that the sound waves can only travel through the $40^{\circ}$-wide opening. Four observation points are located at positions $\vec{x}_{1}=[0.5,0] \mathrm{m}$,

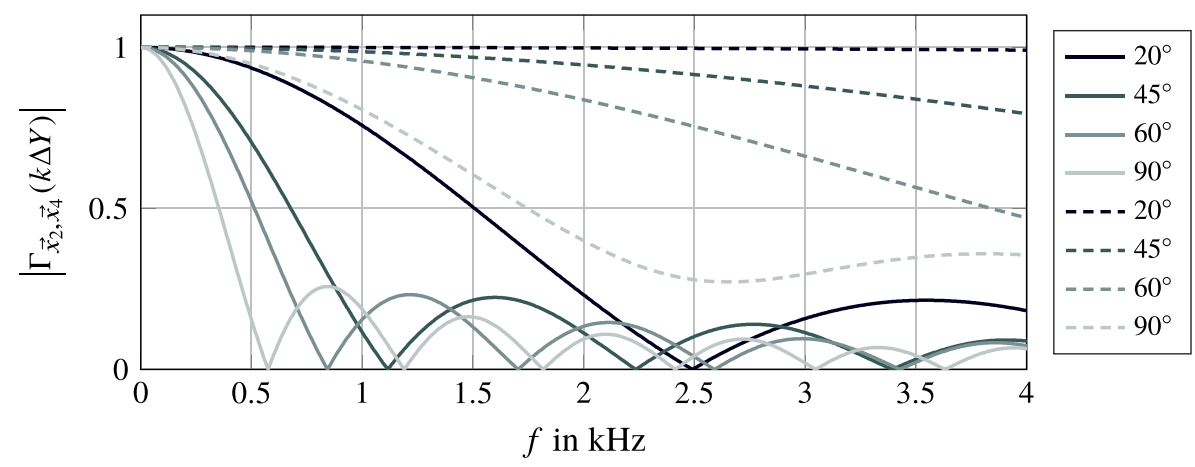

FIG. 4. (Color online) The solid lines show the analytically computed spatial coherence between two observations points $\vec{x}_{2}$ and $\vec{x}_{4}$ separated by $\Delta Y=0.4 \mathrm{~m}$ as resulting from a continuous distribution of line sources of different angular extensions $\Delta \phi_{y} \in\left\{20^{\circ}, 45^{\circ}, 60^{\circ}, 90^{\circ}\right\}$ with radius $R=3 \mathrm{~m}$ and uncorrelated weights $g_{m}$ of unit power. For comparison, the dashed lines show the spatial coherence $\Gamma_{\vec{x}_{1} \vec{x}_{3}}(k \Delta X)$ for observation points $\vec{x}_{1}$ and $\vec{x}_{3}$ separated by $\Delta X=0.4 \mathrm{~m}$ along the $x$ axis (see Fig. 2). 


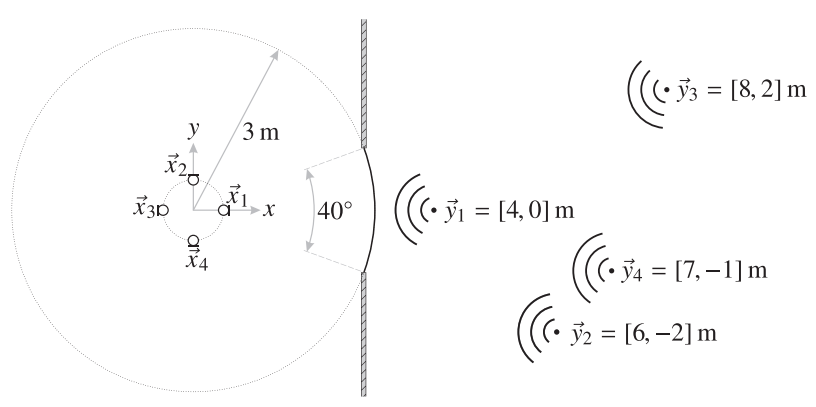

FIG. 5. Illustration of the simulation setup with a spatially windowed noise field evoked by four uncorrelated white Gaussian noise sources located at the indicated positions and observation points at the following $[x, y]$ coordinates: $\vec{x}_{1}=[0.5,0] \mathrm{m}, \vec{x}_{2}=[0,0.5] \mathrm{m}, \vec{x}_{3}=[-0.5,0] \mathrm{m}$, and $\vec{x}_{4}=[0,-0.5] \mathrm{m}$.

$\vec{x}_{2}=[0,0.5] \mathrm{m}, \vec{x}_{3}=[-0.5,0] \mathrm{m}$, and $\vec{x}_{4}=[0,-0.5] \mathrm{m}$, i.e., they are uniformly distributed around a circle of radius $r=0.5 \mathrm{~m}$ with an angular spacing of $90^{\circ}$. To describe the diffraction effects due to the opening and the resulting noise field in the interior, the Huygens-Fresnel principle is applied. For this purpose, the sound field is sampled on the arc with an angular resolution of $0.5^{\circ}$, where an elementary line source is placed at each of the sampling points.

The magnitudes of the resulting spatial coherence functions $\Gamma_{\vec{x}_{1}, \vec{x}_{3}}$ and $\Gamma_{\vec{x}_{2}, \vec{x}_{4}}$ for the horizontal and vertical microphone pairs, respectively, are shown in Fig. 6. Illustrated are the coherence curves resulting from the Welch method in comparison to the coherence based on Eq. (21), where different values for the maximum order $\breve{M} \in\{5,10,20\}$ of the excitation modes $B_{m}$ are evaluated. It can be seen in the left column that the coherence $\Gamma_{\vec{x}_{1}, \vec{x}_{3}}$ obtained with the proposed model for $\breve{M}=5$ already matches the Welch-based coherence estimate very well, and an increase of $\breve{M}$ has virtually no effect. For the coherence $\Gamma_{\vec{x}_{2}, \vec{x}_{4}}$ plotted in the right column, however, there are severe deviations between the results obtained with the Welch method and the ones from the proposed modal description with $\breve{M}=5$, even though the distances between the respective observation points are identical in both cases. These deviations are especially pronounced for higher frequencies. An increase of the maximum order of utilized excitation modes to $\breve{M}=10$ significantly reduces the deviations, and the coherence curves match well for $\breve{M}=20$. The coherence curves for $\breve{M}>20$ are not shown here as they are very similar to $\breve{M}=20$.

Figure 6 reveals that the number of excitation modes $B_{m}$ which is required to precisely describe the sound field varies strongly with the positions of the observation points. More precisely, a lower number of $\breve{M}$ is sufficient for the pair of observation points whose connection line points towards the opening, whereas larger values of $\breve{M}$ are required for the second pair of observation points oriented perpendicularly. This

$$
\left|\Gamma_{\vec{x}_{1}, \vec{x}_{3}}\left(k\left\|\vec{x}_{1}-\vec{x}_{3}\right\|_{2}\right)\right|
$$

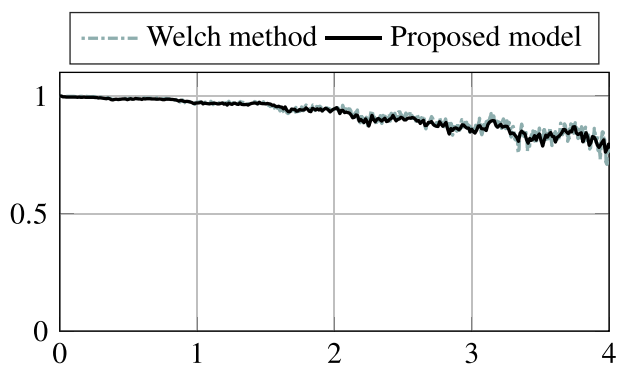

(a) $\breve{M}=5$

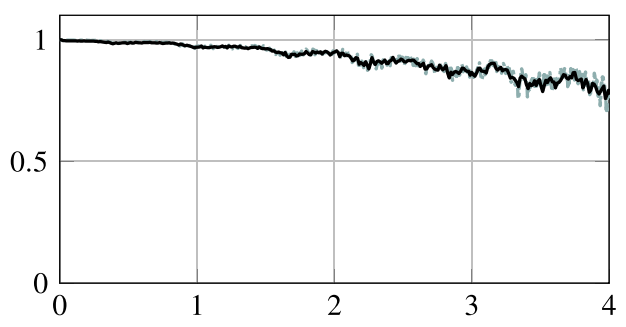

(c) $\breve{M}=10$

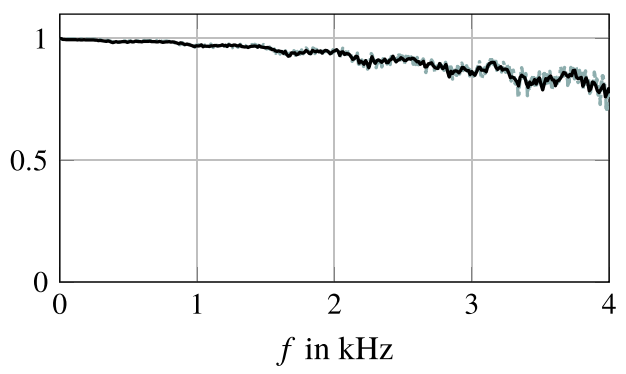

(e) $\breve{M}=20$

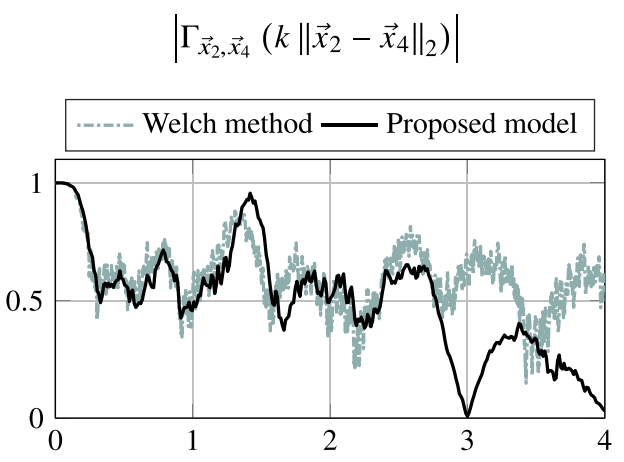

(b) $\breve{M}=5$

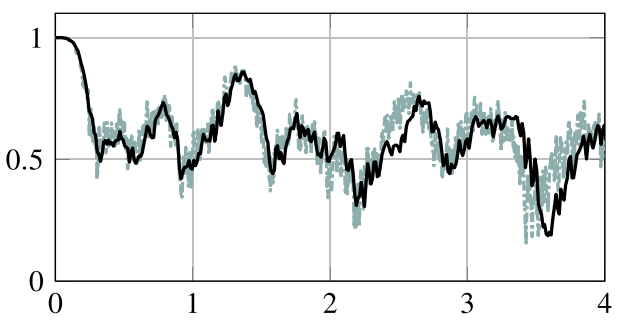

(d) $\breve{M}=10$

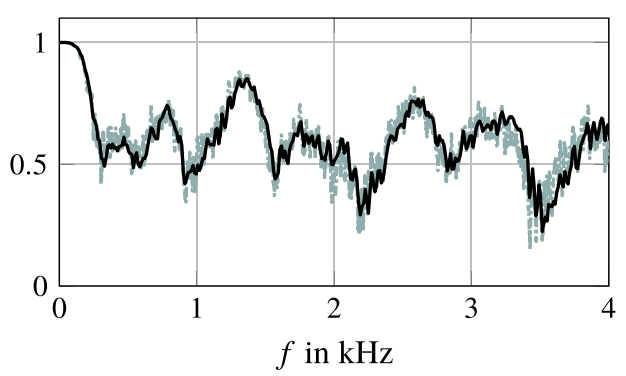

(f) $\breve{M}=20$
FIG. 6. (Color online) Magnitudes of the spatial coherence $\Gamma_{\vec{x}_{1}, \vec{x}_{3}}$ (left column) and $\Gamma_{\vec{x}_{2}, \vec{x}_{4}}$ (right column) for two pairs of observation points, where the underlying setup is illustrated in Fig. 5. The plots show a comparison of the results obtained using the Welch method and the proposed model with a different maximum order $\breve{M}$ of considered spatial basis functions $B_{m}$. 
can be explained by the fact that the wave fronts in the region of interest travel mainly from the right to the left, where the coherence along the propagation direction does not change significantly. In contrast, the sound field may vary strongly in the vertical direction, which is captured by the pair of observation points oriented perpendicularly to the main propagation direction. This behavior is similar to the one already observed in the first simulation (see Fig. 4) and is again related to the trace wavenumber.

\section{CONCLUSIONS AND OUTLOOK}

This contribution provides a general analytic description for the spatial coherence of noise fields evoked by a given pressure distribution on a cylindrical or spherical contour surrounding the region of interest. According to the Huygens-Fresnel principle, the sound pressure on these contours has been represented by a continuous distribution of elementary line or point sources. To model the spatial characteristics of the source distribution on the contour, orthogonal excitation modes have been utilized, where a potential cross-correlation between different modes has been taken into account. Based on this representation, a connection between the cross-correlation of the excitation modes on the contour and the spatial coherence of the resulting noise field in the interior has been established for the free-field case and source distributions of arbitrary angular extensions. Furthermore, the well-known expressions for the coherence of cylindrically isotropic and diffuse noise fields have been derived using the modal representation. By means of different simulated noise fields, the presented analytic expressions for the spatial coherence have been verified and compared with estimates based on the Welch method.

It has been shown that the derived relations allow for the computation of the spatial coherence for known source distributions at any pair of observation points in the free space, while no sensors are required at the same. Thereby, an upper bound for the performance of an ANC system can be predicted at any position in the region of interest. In order to assess the maximum possible performance of global ANC for real scenarios, it is necessary to gain insights into the behavior of real noise sources, i.e., understanding the shape and number of active excitation modes as well as the crosscorrelation between them, which is a topic for further research. Therefore, this work may be seen as the first steps towards a possibly compact or even sparse representation of spatially extended noise sources, which could be especially beneficial when aiming at efficient and global ANC.

\section{ACKNOWLEDGMENT}

This work was supported by the Australian Research Council (ARC) Discovery Projects funding scheme under Project No. DP140103412.

${ }^{1}$ P. D. Teal, T. D. Abhayapala, and R. A. Kennedy, "Spatial correlation for general distributions of scatterers," IEEE Sign. Process. Lett. 9(10), 305-308 (2002).

${ }^{2}$ T. Betlehem and T. D. Abhayapala, "Spatial correlation for correlated scatterers," in IEEE International Conference on Acoustics, Speech, and Signal Processing (ICASSP), Toulouse, France (2006), pp. 5-8.
${ }^{3} \mathrm{H}$. Cox, "Line array performance when the signal coherence is spatially dependent," J. Acoust. Soc. Am. 54(6), 1743-1746 (1973).

${ }^{4}$ H. Buchner, R. Aichner, and W. Kellermann, "TRINICON: A versatile framework for multichannel blind signal processing," in IEEE International Conference on Acoustics, Speech, and Signal Processing (ICASSP), Montreal, Canada (May 2004).

${ }^{5}$ S. Makino, T.-W. Lee, and H. Sawada, Blind Speech Separation (Springer, Dordrecht, the Netherlands, 2007).

${ }^{6}$ J. B. Allen, D. A. Berkley, and J. Blauert, "Multimicrophone signalprocessing technique to remove room reverberation from speech signals," J. Acoust. Soc. Am. 62(4), 912-915 (1977).

${ }^{7}$ P. Naylor and N. D. Gaubitch, Speech Dereverberation (Springer, London, 2010).

${ }^{8}$ A. Schwarz and W. Kellermann, "Coherent-to-diffuse power ratio estimation for dereverberation," IEEE Trans. Audio Speech Lang. Process. 23(6), 1006-1018 (2015).

${ }^{9}$ G. Pinton, G. Trahey, and J. Dahl, "Spatial coherence in human tissue: Implications for imaging and measurement," IEEE Trans. Ultrason. Ferroelectr. Freq. Control 61(12), 1976-1987 (2014).

${ }^{10}$ H. F. Olson and E. G. May, "Electronic sound absorber," J. Acoust. Soc. Am. 25(6), 1130-1136 (1953).

${ }^{11}$ J. C. Burgess, "Active adaptive sound control in a duct: A computer simulation,” J. Acoust. Soc. Am. 70(3), 715-726 (1981).

${ }^{12}$ S. J. Elliott and P. A. Nelson, “Active noise control," IEEE Sign. Process. Mag. 10(4), 12-35 (1993).

${ }^{13}$ S. Kuo, Active Noise Control Systems: Algorithms and DSP Implementations (Wiley, New York, 1996), p. 57.

${ }^{14}$ Y. Kajikawa, W.-S. Gan, and S. M. Kuo, "Recent advances on active noise control: Open issues and innovative applications," APSIPA Trans. Sign. Inf. Process. 1(e3), 1-21 (2012).

${ }^{15}$ P. Samarasinghe, W. Zhang, and T. D. Abhayapala, "Recent advances in active noise control inside automobile cabins: Toward quieter cars," IEEE Signal Process. Mag. 33(6), 61-73 (2016).

${ }^{16}$ J. Zhang, T. D. Abhayapala, P. N. Samarasinghe, W. Zhang, and S. Jiang, "Multichannel active noise control for spatially sparse noise fields," J. Acoust. Soc. Am. 140(6), EL510-EL516 (2016).

${ }^{17}$ F. Jacobsen and T. Roisin, "The coherence of reverberant sound fields," J. Acoust. Soc. Am. 108(1), 204-210 (2000).

${ }^{18}$ M. Kuster, "Spatial correlation and coherence in reverberant acoustic fields: Extension to microphones with arbitrary first-order directivity," J. Acoust. Soc. Am. 123(1), 154-162 (2008).

${ }^{19}$ S. Nees, A. Schwarz, and W. Kellermann, "A model for the temporal evolution of the spatial coherence in decaying reverberant sound fields," J. Acoust. Soc. Am. 138(3), EL248-EL253 (2015).

${ }^{20}$ H. Kuttruff, Room Acoustics (Taylor and Francis, New York, 2009).

${ }^{21}$ O. Thiergart, G. Del Galdo, and E. A. Habets, "On the spatial coherence in mixed sound fields and its application to signal-to-diffuse ratio estimation,” J. Acoust. Soc. Am. 132(4), 2337-2346 (2012).

${ }^{22}$ Y. A. Huang, A. Luebs, J. Skoglund, and W. B. Kleijn, "Globally optimized least-squares post-filtering for microphone array speech enhancement," in IEEE International Conference on Acoustics, Speech, and Signal Processing (ICASSP), Shanghai, China, 2016.

${ }^{23}$ Y. J. Wu and T. D. Abhayapala, "Theory and design of soundfield reproduction using continuous loudspeaker concept," IEEE Trans. Audio Speech Lang. Process. 17(1), 107-116 (2009).

${ }^{24}$ M. Zollner, Physik der Elektrogitarre (The Physics of Electric Guitars) (Eigenverlag, Regensburg, Germany, 2009).

${ }^{25}$ H.-Y. Chiang and Y.-H. Huang, "Vibration and sound radiation of an electrostatic speaker based on circular diaphragm," J. Acoust. Soc. Am. 137(4), 1714-1721 (2015).

${ }^{26} \mathrm{H}$. Cox, "Spatial correlation in arbitrary noise fields with application to ambient sea noise," J. Acoust. Soc. Am. 54(5), 1289-1301 (1973).

${ }^{27}$ M. Schneider and W. Kellermann, "A wave-domain model for acoustic mimo systems with reduced complexity," in IEEE Workshop on HandsFree Speech Communication, Microphone Arrays (HSCMA), Edinburgh, UK (2011).

${ }^{28}$ H. Chen, P. Samarasinghe, T. D. Abhayapala, and W. Zhang, "Spatial noise cancellation inside cars: Performance analysis and experimental results," in IEEE Workshop Applications of Signal Processing Audio, Acoustics (WASPAA), New Paltz (2015), pp. 1-5.

${ }^{29}$ D. P. Jarrett, E. A. Habets, and P. A. Naylor, Theory and Applications of Spherical Microphone Array Processing (Springer Topics in Signal Processing) (Springer, Basel, Switzerland, 2016). 
${ }^{30}$ E. G. Williams, Fourier Acoustics: Sound Radiation and Nearfield Acoustical Holography (Academic, London, UK, 1999), pp. 192 [Eq. (6.48)], 253 [Eq. (8.5)], 266 [Eq. (8.47)].

${ }^{31}$ D. Colton and R. Kress, Inverse Acoustic and Electromagnetic Scattering Theory (Springer, New York, 2013), Vol. 93, pp. 26 [Eq. (2.28)], 31 [Eqs. (2.42) and (2.43)], 75 [Eq. (3.88)].

${ }^{32}$ R. Kennedy, P. Sadeghi, T. D. Abhayapala, and H. M. Jones, "Intrinsic limits of dimensionality and richness in random multipath fields," IEEE Trans. Sign. Process. 55(6), 2542-2556 (2007).

${ }^{33} \mathrm{M}$. Abramowitz and I. A. Stegun, Handbook of Mathematical Functions With Formulas, Graphs, and Mathematical Tables (U.S. Department of Commerce, National Bureau of Standards, 1964), pp. 363 [Eq. (9.1.79)], 364 [Eq. (9.2.4)].
${ }^{34}$ R. K. Cook, R. V. Waterhouse, R. D. Berendt, S. Edelman, and M. C. Thompson, "Measurement of correlation coefficients in reverberant sound fields," J. Acoust. Soc. Am. 27(6), 1072-1077 (1955).

${ }^{35}$ D. Slepian and H. O. Pollak, "Prolate spheroidal wave functions, Fourier analysis, and uncertainty-I," Bell Syst. Tech. J. 40(1), 43-63 (1961).

${ }^{36}$ F. J. Simons, "Slepian functions and their use in signal estimation and spectral analysis," in Handbook of Geomathematics (Springer, Berlin, 2010), pp. 891-923.

${ }^{37}$ P. A. Martin, Multiple Scattering-Interaction of Time-Harmonic Waves with N Obstacles (Cambridge University Press, Cambridge, 2006), p. 80 [Eq. (3.61)]. 